\title{
BIO.I I - Establishment and evaluation of a transient expression method in Expi293F system for Fab monoclonal antibody fragment production
}

Felipe Rodrigues Semcovici Ramos ${ }^{1 *}$; Anna Erika Vieira de Araújo'; Haroldo Cid da Silva Júnior'; José Procópio Moreno Senna ${ }^{1}$.

1Fiocruz/Bio-Manguinhos.

Introduction: Monoclonal antibodies and their fragments produced by recombinant DNA technology represent a group of immunobiologicals with varied applications in immunotherapy and as a tool for immunodiagnostics assays development. Expression of these proteins require efficient cell culture systems that ensure agility in protein synthesis, acceptable benefit-cost ratios, high yields and expression of functional proteins for the desired applications.

Objective: The study objective was to establish a method for transient expression of Fab monoclonal antibodies fragments in eukaryotes cell cultures (Expi293F system), in order to attend strategic demands for implementation of a recombinant antibody fragments expression platform.

Methodology: The genetic constructs required for the expression of the Fab light and Fd chains monomers were produced by amplifying the respective coding regions, digesting them and ligating the inserts to pCI-neo and pCDNA3.4 plasmid vectors. The selection of the recombinant clones was performed in E. coli cells, with subsequent restriction enzymes digestion to analyze the correct orientation of the inserts. HEK 293 cells were grown in suspension at $37^{\circ} \mathrm{C}, 120 \mathrm{RPM}$, $8 \% \mathrm{CO}_{2}$ and transfected with the recombinant constructs by using liposomal vesicles as DNA carrier. After transfection, the cell cultures were followed during seven days to assess the kinetics of cell growth and viability. Supernatant aliquots were daily taken to analyze Fab expression by Western Blot (WB) and SDS-PAGE in non-reducing conditions. Fab fragment quantification was conducted by immunoenzymatic assay specific design for Fab capture.

Results: The light and Fd genetic sequences amplification and cloning process allowed to obtain four recombinant genetic constructs with correct orientation of the inserts into the expression vectors. The cell growth and viability kinetics assay demonstrated similar profiles for the cell cultures transfected with the genetic constructs in both expression vectors tested. The WB analysis indicated a gradual increase on the intensity of the Fab band along cell culture cultivation. In SDS-PAGE analysis, a visual difference on the Fab band was observed between the supernatant samples, with higher intensity of the bands in the samples collected from the cell cultures transfected with the genetic constructs in pCDNA3.4 vector. ELISA quantification demonstrated Fab expression 2000 times higher in cell cultures transfected with the genetic constructs in pCDNA3.4 vector, resulting in final protein levels of $0,8 \mathrm{mg} / \mathrm{mL}$.

Conclusion: The experimental data collected from this study identified the suitability of the Expi293F system as a plataform for expression of recombinant antibody fragments. Fab levels obtained with the genetic constructs in pCDNA3.4 vector were higher than those described in the literature for expression in HEK cells. Furthermore, the registered expression levels were higher than other systems usually applied for expression of recombinant antibody fragments. Therefore, this model can be employed to meet pre-clinical research and development demands for these types of biomolecules.

Keywords: Fab monoclonal antibody fragment; recombinant protein transient expression; Expi293F system 